\title{
Surface photochemistry: benzophenone as a probe for the study of silica and reversed-phase silica surfaces $\dagger$
}

\author{
L. F. Vieira Ferreira, ${ }^{* a}$ I. Ferreira Machado, ${ }^{a}$ J. P. Da Silva ${ }^{a, b}$ and T. J. F. Branco ${ }^{a}$ \\ Received 11th January 2006, Accepted 27th March 2006 \\ First published as an Advance Article on the web 7th April 2006 \\ DOI: $10.1039 / \mathrm{b600384b}$
}

This work reports the use of benzophenone, a very well characterized probe, to study new hosts: two reversed-phase silicas. Laser-induced room temperature luminescence of argon purged solid powdered samples of benzophenone adsorbed onto the two different reversed-phase silicas, RP-18 and RP-8, revealed the existence of a low energy emission band in contrast with the benzophenone adsorbed on $60 \AA$ A pore silica, where only triplet benzophenone emits. This low energy emission band was identified as the fluorescence of the ketyl radical of benzophenone, which is formed as the result of a hydrogen atom abstraction reaction of the triplet excited benzophenone from the alkyl groups of the surface of the reversed silicas. Such emission does not exist for benzophenone adsorbed onto $60 \AA$ pore silica. Room temperature phosphorescence was obtained in argon purged samples for all the surfaces under use. The decay times of the benzophenone emission vary greatly with the alkylation of the silica surface when compared with "normal" silica surface. A lifetime distributions analysis has shown that the shortest lifetimes for the benzophenone emission exist in the former case. Triplet-triplet absorption of benzophenone was detected in all cases and is the predominant absorption in the case of $60 \AA$ pore silica, while benzophenone ketyl radical formation occurs in the case of the reversed silicas. Diffuse reflectance laser flash photolysis and gas chromatography-mass spectrometry techniques provided complementary information, the former about transient species and the latter regarding the final products formed after laser irradiation, both at $266 \mathrm{~nm}$ or $355 \mathrm{~nm}$. Product analysis and identification show that the degradation photoproducts are dependent on the excitation wavelength, the photochemistry being much more rich and complex in the $266 \mathrm{~nm}$ excitation case, where an $\alpha$-cleavage reaction occurs. A detailed mechanistic analysis is proposed.

\section{Introduction}

Time resolved laser induced luminescence, diffuse reflectance laser flash-photolysis and ground state diffuse reflectance absorption spectroscopy are relatively new techniques that can be applied to study opaque and crystalline systems. ${ }^{1-8}$ These solid state photochemical methods have been recently applied by our group to study several organic compounds adsorbed onto different hosts such as $p$-tert-butylcalix[4], [6] and [8]arenes and derivatives, ${ }^{9-11}$ microcrystalline cellulose, ${ }^{12-14}$ silicalite and cyclodextrins, ${ }^{15-17}$ silica $^{18}$ and resorcinarene frameworks, ${ }^{19}$ among others.

Benzophenone (BZP) is an extremely useful molecule for probing new hosts. The $\mathrm{n} \rightarrow \pi^{*}$ absorption transition was found to be very sensitive to the environment characteristics and also exhibits a photochemistry which depends on the host properties. ${ }^{9-10,12,20}$ In a very recent paper ${ }^{20}$ we reported a comparative study of the luminescent properties of BZP adsorbed onto a mesoporous channel-type solid support material called MCM-41 with other nano-porous materials, namely silicalite (a de-aluminated zeolite)

${ }^{a}$ Centro de Química-Física Molecular-Complexo Interdisciplinar, Instituto Superior Técnico, Av. Rovisco Pais, 1049-001, Lisboa, Portugal. E-mail: LuisFilipeVF@ist.utl.pt

${ }^{b}$ FCT, Universidade do Algarve, Campus de Gambelas, 8005-139, Faro, Portugal

$\dagger$ Electronic supplementary information (ESI) available: Fig. S1 and S2. See DOI: $10.1039 / \mathrm{b} 600384 \mathrm{~b}$ and H-ZSM-5 (with an acidic internal surface). Triplet state luminescence was observed in all cases, hydrogen bonded excited benzophenone was detected on MCM-41 while protonated excited BZP luminescence was observed only on H-ZSM-5. Time resolved diffuse reflectance absorption technique was also applied to the powdered solid samples of these compounds proving undoubtedly the inclusion of BZP within the cavities of these three hosts, and the existence of triplet state of BZP in air equilibrated samples in all cases, following laser excitation. $\mathrm{BZP} \mathrm{P}^{\bullet} \mathrm{OH}$ radical was also detected and is one of the important intermediates.

Reversed-phase silicas are used in reversed-phase chromatography, where the stationary phase is non-polar (often a hydrocarbon) and the mobile phase is a relatively polar or polar solvents such as methanol or acetonitrile and water, or a mixture of them. ${ }^{21}$ These silicas have octyl- or octadecyl siloxane groups on the silica surface (the silanol groups of the "normal" silica surface $\equiv \mathrm{SiOH}$ react with $\mathrm{Cl}\left(\mathrm{CH}_{3}\right)_{2} \mathrm{Si}-\mathrm{R}$ in acidic hot media, forming the organosiloxane, $\equiv \mathrm{SiO}-\left(\mathrm{CH}_{3}\right)_{2} \mathrm{Si}-\mathrm{R}$ and $\mathrm{HCl}$, where $\mathrm{R}=$ octylor octadecyl-groups). These long hydrocarbon groups are aligned parallel to one anther and perpendicular to the surface of the particle, forming a brush-like, non-polar silica surface. ${ }^{21}$ Many successful partition chromatography separations were achieved with the use of these materials.

This work reports the use of benzophenone, a very well characterized probe, to study new hosts (two reversed-phase silicas). In these hosts all surface silanols were replaced by alkylsiloxane 
groups with alkyl chains with eighteen or eight carbon atoms, therefore transforming the polar surface of the normal silicas into surfaces with a non-polar character. A comparison of the photochemical behaviour of BZP was made with $60 \AA$ pore size silica.

Experimental evidence will be presented for the first time, showing the occurrence of a photochemical reaction of benzophenone on the surface of the reversed-phase silica, resulting in the formation of a new species, the diphenylketyl radical, which is also excited within a single laser pulse and emits its own luminescence.

A detailed chromatographic study of the laser (and lamp) irradiated samples was made, which provided experimental results pointing to a clear dependence of the photochemistry on the excitation wavelength under use.

\section{Experimental}

\section{Materials}

Silicalite (Union Carbide) and $60 \AA$ pore silica gel (Merck, 70230 mesh) were used as powdered solid supports as received. Benzophenone (Koch-Light, Scintillation grade) and the solvents hexane, dichloromethane, iso-octane, methanol and acetonitrile were from Merck, LiChrosolv grade. These compounds were first analysed by chromatography before use.

RP-18 and RP-8 were purchased from Merck (LiChrosorb grade) and used as received. The specific surface areas indicated by the producer are 150 and $250 \mathrm{~m}^{2} \mathrm{~g}^{-1}$ respectively.

\section{Sample preparation}

The samples used in this work were prepared using the solvent evaporation method. This method consists in the addition of a solution containing the probe to the previously dried powdered solid substrate, followed by solvent evaporation from the stirred slurry in a fume cupboard. Hexane or dichloromethane were used for sample preparation in the case of the $60 \AA$ silica and for the reversed-phase silicas because of the high solubility of BZP in these solvents and also because these solvents evaporate easily at room temperature in the fume cupboard. In the case of the $\mathrm{BZP} /$ silicalite samples iso-octane was used for BZP inclusion into the channels of the adsorbent, because the solvent molecule is not able to penetrate into the channels due to its molecular dimensions. The final solvent removal was performed overnight in an acrylic chamber with an electrically heated shelf (Heto, Model FD 1.0110) with temperature control $\left(30 \pm 1{ }^{\circ} \mathrm{C}\right)$ and under moderate vacuum at a pressure of $c a$. $10^{-3}$ Torr.

Solvent evaporation in the fume cupboard was done in the dark. All samples are kept in the dark before the experiments are performed. The experiments are also performed in dark conditions so that only the light arising from the excitation sources (lamps and lasers) reaches the samples under study.

\section{Methods}

1. Ground state diffuse reflectance absorption spectra (GSDR). Ground state absorption spectra for the solid samples were recorded using an OLIS 14 spectrophotometer with a diffuse reflectance attachment. Further details are given elsewhere. ${ }^{1,12-14}$

2. Laser induced luminescence (LIL) and diffuse reflectance laser flash photolysis (DRLFP) systems. Schematic diagrams of the LIL and of the DRLFP systems are presented in ref. 1 and 16. Laser flash photolysis experiments were carried out with the third or the fourth harmonic of a Nd:YAG laser $(355$ and $266 \mathrm{~nm}$, ca. $6 \mathrm{~ns}$ FWHM, $\sim 10-30 \mathrm{~mJ}$ pulse ${ }^{-1}$ ) from B. M. Industries (Thomson-CSF, model Saga 12-10), in the diffuse reflectance mode. The light arising from the irradiation of solid samples by the laser pulse is collected by a collimating beam probe coupled to an optical fiber (fused silica) and is detected by a gated intensified charge coupled device Oriel model Instaspec V, (Andor ICCD, based on the Hamamatsu S57 69-0907). The ICCD is coupled to a fixed imaging compact spectrograph (Oriel, model FICS 77441). The system can be used either by capturing all light emitted by the sample or in a time-resolved mode by using a delay box (Stanford Research Systems, model D6535). The ICCD has high speed gating electronics (2.2 ns) and intensifier and covers the 200-900 nm wavelength range. Time-resolved absorption and emission spectra are available in the nanosecond to second time range. Transient absorption data are reported as percentage of absorption ( $\% \mathrm{Abs}$.), defined as $100 \Delta J_{t} / J_{\mathrm{o}}=\left(1-J_{t} / J_{\mathrm{o}}\right) 100$, where $J_{\mathrm{o}}$ and $J_{t}$ are diffuse reflected light from sample before exposure to the exciting laser pulse and at time $t$ after excitation, respectively. For laser-induced luminescence experiments with a $\mathrm{N}_{2}$ laser (PTI model 2000, $c a$. 600 ps FWHM, $\sim 1.1 \mathrm{~mJ}$ pulse $^{-1}$ ), was also used. In this case the excitation wavelength is $337 \mathrm{~nm}$. With these set-ups, both fluorescence and phosphorescence spectra are easily available (by the use of the variable time gate width and start delay facilities of the ICCD).

3. Irradiation and product analysis. Photodegradation studies under lamp irradiation were conducted in a reactor previously used to study the photochemistry of several compounds at the solid/gas interface. ${ }^{22,23}$ Lamp irradiation was performed at $254 \mathrm{~nm}$ using a $16 \mathrm{~W}$ low-pressure mercury lamp (Applied Photophysics) without filters and without refrigeration and a xenon lamp with a glass filter. The samples were irradiated in a quartz cell placed at $1 \mathrm{~cm}$ from the lamp surface, in air equilibrated conditions, during $3.5 \mathrm{~h}$. Laser irradiation at $355 \mathrm{~nm}$ and $266 \mathrm{~nm}$, in argon atmosphere, was also performed. In this case the samples were irradiated in a quartz cell during $0.5 \mathrm{~h}$ at five pulses $(30 \mathrm{~mJ})$ per second. The samples were mixed every $5 \mathrm{~min}$ during the laser irradiation process. Non-irradiated and irradiated samples were analysed after extraction with acetonitrile (a known weight of sample in a known volume of solvent) followed by centrifugation. Photolysis was followed by HPLC using a Merck-Hitachi 655A11 chromatograph equipped with detectors 655A-22 UV and Shimadzu SPD-M6A photodiode array. A column LiChroCART 125 (RP-18, $5 \mu \mathrm{m}$ ) Merck was used and the runs were performed using mixtures water-acetonitrile. The extracts were also analysed by GC-MS using a Hewlett Packard 5890 Series II gas chromatograph with a 5971 series mass selective detector (E.I. $70 \mathrm{eV}$ ). A Restek RTX-20 capillary column with $20 \mathrm{~m}$ and $0.18 \mathrm{~mm}$ id was used. The initial temperature $70^{\circ} \mathrm{C}$ was maintained during $5 \mathrm{~min}$ and then a rate of $5{ }^{\circ} \mathrm{C} \mathrm{min}^{-1}$ was used up to $250{ }^{\circ} \mathrm{C}$. 


\section{Results and discussion}

\section{Ground state diffuse reflectance absorption spectra}

Ground state diffuse reflectance absorption spectra for BZP adsorbed onto RP-18, RP-8, $60 \AA$ pore silica and silicalite, were obtained with the use of an integrating sphere. ${ }^{1,12-14}$

The BZP ground state absorption $\mathrm{S}_{0} \rightarrow \mathrm{S}_{1}$ transition $(\mathrm{n} \rightarrow$ $\left.\pi^{*}\right)$ has an absorption maximum at about $347 \mathrm{~nm}$ for silicalite as host material and a clear vibrational structure characteristic of the carbonyl group in the excited state ${ }^{24}$ can be observed when BZP is included into the rigid channel structure of this adsorbent (see Fig. S1, in the electronic supplementary information, ESI). $\dagger$

Silicalite is a de-aluminated analogue of ZSM-5 zeolite, with straight and zig-zag channels interconnected. Due to the lack of substitutional aluminium, this adsorbent has no catalytic or exchange properties when compared to the ZSM-5 zeolites. Silicalite is the only known hydrophobic form of silica and is capable of adsorbing organic molecules up to about $6 \AA$ of kinetic diameter, even removing them from water. It provides a very rigid and hydrophobic environment for the BZP adsorption inside both channels ${ }^{25}$ and this is reflected in the vibrational structure of both absorption and emission spectra. ${ }^{15}$

In the case of the other three surfaces, RP-18, RP-8 and $60 \AA$ silica (all curves were normalized at $315 \mathrm{~nm}$ and the BZP concentration was $250 \mu \mathrm{mol} \mathrm{g} \mathrm{g}^{-1}$ ), the absorption curves appear now more and more as broad bands, shifted to the blue in that order. These hypsochromic shifts are quite characteristic of the $n \rightarrow \pi^{*}$ transition with increasing polarity of the surface. ${ }^{12,15,20}$ The broadening of the spectra is probably related to both heterogeneity of the adsorbent and a much smaller rigidity of the adsorbed probe. Solution absorption spectra of BZP, for instance in cyclohexane and ethanol, also exhibit this type of influence of polarity, characteristic of the $\mathrm{n} \rightarrow \pi^{*}$ transition. ${ }^{24}$

Clearly, going from RP-18 to RP-8 and to $60 \AA$ silica, an increase in the surface polarity is observed, quite consistent with the surface characteristics: long alkyl chains with 18 carbons in RP-18, only 8 carbons in RP-8 and contact with the hydroxyl groups of the surface silanols in the case of $60 \AA$ silica.

\section{Room temperature laser induced phosphorescence}

Fig. 1 presents the room temperature phosphorescence spectra of BZP onto the surfaces of the $60 \AA$ pore silica gel (part a) and of the RP-18 reversed-phase silica (part b).

Similar luminescence data for BZP adsorbed on silicalite and included into the cavities of calixarenes are presented in ref. 15 and 9, 10 respectively. Those time resolved spectra were obtained with air equilibrated conditions and were identical to the ones obtained with argon purged samples. Lifetimes of about $80 \mu \mathrm{s}$ were determined for the calixarene inclusion ${ }^{9-11}$ and $3.1 \mathrm{~ms}$ for inclusion into the narrower channels of silicalite, ${ }^{25}$ as compared to about $50 \mu$ s for benzophenone microcrystals, all determined at the maximum emission wavelength (about $448 \mathrm{~nm}$ ). ${ }^{12}$

The luminescence spectrum of benzophenone adsorbed onto reversed-phase silica surface does not present a rise time in a nanosecond timescale, as Fig. 1a shows. Other time resolved spectra in a 1-10 ns timescale were recorded (data not presented in the paper) and confirm this behaviour.
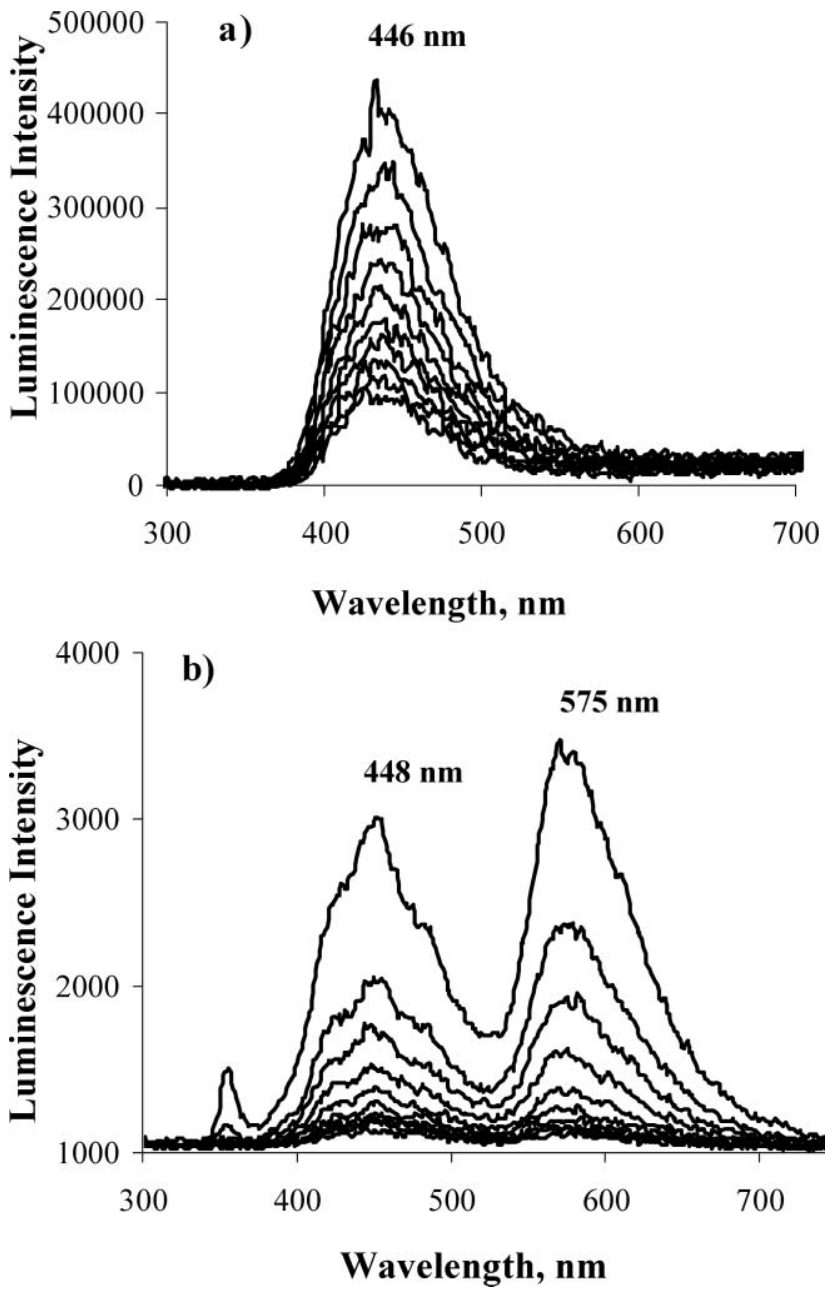

Fig. 1 a) Laser induced phosphorescence spectra for argon purged samples of benzophenone adsorbed onto 60 Å pore silica. The excitation wavelength was $337 \mathrm{~nm}$ and curves were recorded every $1 \mathrm{~ms}$ after the laser pulse $\left(t_{0}=1 \mathrm{~ms}\right)$. b) Laser induced phosphorescence spectra for argon purged samples of benzophenone adsorbed onto reversed-phase silica (RP-18). The excitation wavelength was $355 \mathrm{~nm}$ and curves were recorded every $50 \mathrm{~ns}$ after the laser pulse $\left(t_{0}=0 \mathrm{~ns}\right)$.

It is important to note that the emission of BZP on the silica gel surface peaks at $446 \mathrm{~nm}$ and that this emission is very broad when compared to the emission into the silicalite channels or within the calixarenes cavity, in accordance with the very broad absorption spectrum. The heterogeneity of the adsorbent is also reflected in the wide range of lifetimes determined for this phosphorescence emission of BZP as we will show now.

Recently we have developed a new tool for the lifetime distributions analysis of emissions of probes adsorbed onto heterogeneous surfaces. ${ }^{25}$ This new methodology allows for asymmetric distributions and uses Voigt profiles (Gaussian-Lorentzian product) instead of pure Gaussian or Lorentzian distributions. A very simple and widely available tool for fitting has been used, the Microsoft Excel Solver. This is a very convenient way to treat the emission or transient absorption decay data because it reflects the multiplicity of sites available for the probe onto the specific surface under study. 
A detailed study of the luminescence decay curves of pyrene included within $p$-tert-butylcalix[4]arene cavities and benzophenone into silicalite channels has been reported recently. ${ }^{25}$

The use of the sum of a few exponentials to analyse the decay of probes onto heterogeneous surfaces is a description without physical meaning, ${ }^{25,26}$ and the best tool to study decays of a probe onto heterogeneous surfaces is a lifetime distribution analysis (as Scaiano and co-workers did recently, e.g. ref. 2628). The validation of its conclusions being sustained by other spectroscopic studies.

There was no need here for the use of convolution with the excitation pulse because decay times analysed are in the millisecond or hundred of nanoseconds time domain and the excitation pulse width was 0.6 ns. Modelling studies have shown that for decays with lifetimes greater than $3 \mathrm{~ns}$ the decay curve is not affected by this excitation pulse.

When applied to the BZP/60 ^ silica case (Fig. 2a), and after recording the decay traces within various instrumental timescales (experimental information was obtained starting in the nanosecond timescale and also in the $0.01 \mu$ s or longer timescales), the lifetimes distribution analysis evidences a broad band centred at $0.3 \mu \mathrm{s}$, which we assigned to different conformations of adsorbed BZP due to different adsorption sites of the silica gel surface (the $60 \AA$ size reported for the pore average diameter means that the maximum of the distribution of the pore sizes is at $60 \AA$ but, of course, larger and smaller pores exist, as is clearly indicated for several silica gels in ref. 29), therefore resulting in different conformations for BZP and different emissions. However Fig. 2a also shows a peak at $4.2 \mathrm{~ms}$, showing that some fraction of adsorbed benzophenone molecules is very rigidly adsorbed in

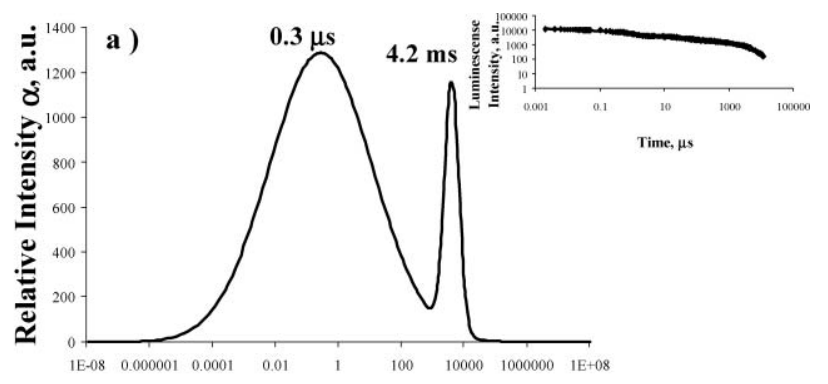

Lifetime, $\mu$ s

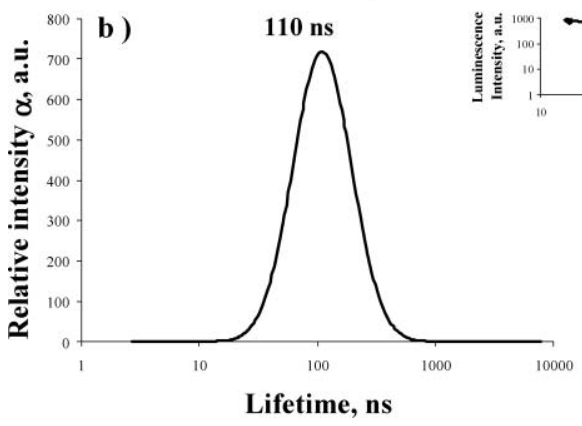

Fig. 2 Lifetime distributions recovered from luminescence decays of argon purged samples of a) benzophenone adsorbed onto $60 \AA$ pore silica, observed at $450 \mathrm{~nm}$; b) benzophenone adsorbed onto reversed-phase silica (RP-18), observed at $450 \mathrm{~nm}$ or $575 \mathrm{~nm}$. The insets show the fitting of the recovered decay superimposed on the experimental data.

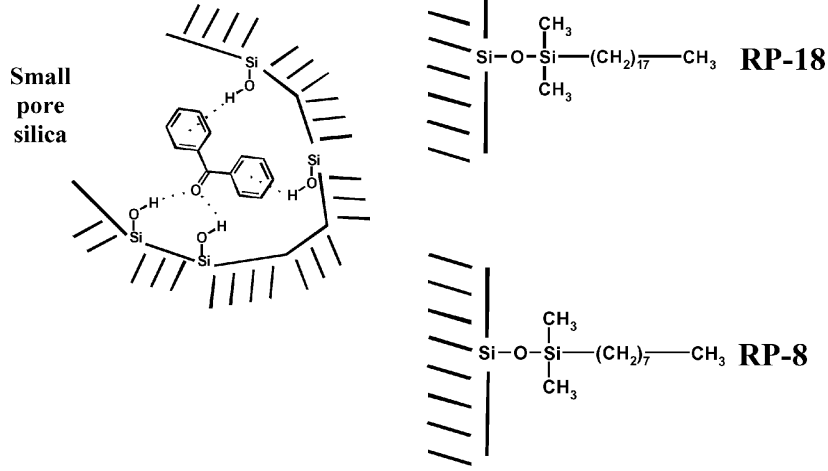

Scheme 1 Schematic representation of the surfaces of a small pore silica and also of RP-18 and RP-8.

some cases and emits with a long lifetime, comparable to the one of silicalite adsorption into the narrower channels. ${ }^{25}$ Some of the pores are particularly suitable for BZP, as the schematic representation presented in Scheme 1 suggests, showing different possibilities for hydrogen bond formation to the silanols of the surface.

Two different time gates were used for the BZP/60 ̊ silica case. Data presented in Fig. 1a were obtained with the use of a very large time gate $(20 \mathrm{~ms}$ width) to record all room temperature phosphorescence. A small time gate (50 or $100 \mathrm{~ns}$ width) was also used to show the short time behaviour immediately after the laser excitation pulse. A special emission of BZP in the nanosecond timescale was detected, very similar to the one reported in Fig. $3 \mathrm{~b}$ of ref. 20 for BZP on MCM-41. This emission peaks at $c a .430 \mathrm{~nm}$, and for these reasons, we assign again this emission to hydrogen bonded BZP emission (data not shown).

In the case of the BZP/RP-18 sample, the use of a small time gate width or large gate width enabled us to observe only one time resolved luminescence spectrum and this means that there is no hydrogen bonded BZP emission in the nanosecond timescale in this case: superimposed on the normal benzophenone phosphorescence emission we detected the appearance of a low energy emission band, which peaks at $575 \mathrm{~nm}$ immediately after the laser pulse. This low energy emission band is shown in Fig. 1 b.

Very similar results (not shown) were obtained for the BZP/RP8 samples.

All experimental luminescence results presented until now for $\mathrm{BZP} / 60 \AA$ silica, BZP/RP-18 and BZP/RP-8 samples were obtained with the use of argon purged samples. Air equilibrated surfaces do not provide detectable emissions or only provide small emissions, showing the oxygen quenching of the excited species is very effective for these powdered solid silica surfaces.

All results at timescales shorter than $6 \mathrm{~ns}$ (laser pulse width) are simply a reflection of the forced shape of the distribution.

In what regards the assignment of the low energy emission band for the BZP/RP-18 or BZP/RP-8 samples two main hypotheses were assumed as starting points: emission from transient species derived from benzophenone alone or from new species resulting from benzophenone photochemical reaction with the host. The second hypothesis seems to be the most logical one. The simple fact that the low energy emission band peaks at $575 \mathrm{~nm}$ (and its lifetime) points to be an emission from the ketyl radical of 
benzophenone which emits at this wavelength range, as the result of a two photon excitation process (fluorescence emission). ${ }^{30-32}$ It also excludes emissions from aryl alkyl ketones which phosphoresces around $420 \mathrm{~nm} .^{16,33,34}$

Previous work indicates that calixarene, ${ }^{9-11}$ cellulose, ${ }^{12-14}$ or $\beta$ cyclodextrin $^{27}$ are good hydrogen atom donors towards BZP (in solid powdered samples), so it sounds reasonable to assume that efficient ketyl radical formation of benzophenone may also occur here.

The lifetime emission distribution for this case is presented in Fig. 2b: only one emissive species is detected (a single distribution of lifetimes peaking at $110 \mathrm{~ns}$ ), with a much shorter lifetime when compared with the BZP emission onto the porous silica. Other important experimental observation is that the same lifetime distribution curve was obtained at $450 \mathrm{~nm}$ or $575 \mathrm{~nm}$, indicating that the lifetime of the ketyl radical emission is the same as the ${ }^{3}$ BZP* emission. From solution data in the literature, we know that the lifetime of the excited diphenylketyl radical is $c a .4 \mathrm{~ns}$ in solvents such as toluene or acetonitrile at room temperature. ${ }^{30}$ How is it possible that on the RP-18 surface this species lives hundreds of nanoseconds?

Two species are emitting on the RP-18 surface, ${ }^{3} \mathrm{BZP} *$ and the excited diphenylketyl radical. The hydrogen atom abstraction from the alkyl chain is competing with the phosphorescence emission and a remarkable quenching effect exists, therefore reducing the lifetime emission from millisecond to about one hundred nanoseconds. This is a much longer lifetime when compared with the excited diphenylketyl radical and this means that this species only has a pseudo lifetime of $110 \mathrm{~ns}$. The emission of the excited diphenylketyl radical is the same as the one of ${ }^{3} \mathrm{BZ} \mathrm{P}^{*}$ because the former excited species is formed from an energy transfer process from ${ }^{3} \mathrm{BZP}$ * and emits in an "instantaneous" manner when compared with the energy donor, therefore in a time resolved spectra, it exhibits the same lifetime of the species which is in its origin. The ketyl radical emission exists with one single laser pulse and could be recorded immediately after that laser pulse. In this case, and taking into account the pulse duration (about 6-7 ns for $355 \mathrm{~nm}$ excitation), the ketyl radical can be formed, excited and also emit during one laser pulse. At later times it fluoresces as soon as it is formed and excited by energy transfer.

This argument for the excitation of the ketyl radical at times larger than the excitation laser pulse, by an energy transfer mechanism (triplet-doublet energy transfer) was reported before for solution studies by Naqvi and Wild ${ }^{31}$ and Netto-Ferreira and Scaiano. ${ }^{30}$ In the solution case a rise time was detected for the ketyl emission. ${ }^{31}$ When BZP is adsorbed onto the reversed-phase silica surface no rise time was detected in the nanosecond timescale. This is due to the confinement of the donor and acceptor species $\left({ }^{3} \mathrm{BZP}^{*}\right.$ and $\mathrm{BZPH}{ }^{*}$ radical are closely entrapped within the long alkyl chains of the reversed-phase silica surface).

The high intense fluorescence band of the diphenylketyl radical is easily detected due to its relatively high fluorescence quantum yield, which is $0.11 \pm 0.02$ in benzene, as reported in ref. 35 .

For low loadings $(\sim 10$ to $50 \mu \mathrm{mol}$ of BZP per gram of the RPS) the ketyl emission is hardly detectable and this is consistent with the fact that the energy transfer mechanism responsible for the observed emission of the excited diphenylketyl radical is concentration dependent.
By keeping the samples in the dark we prevent the possibility of the room light being absorbed, therefore producing ketyl radicals before DRLFP experiments were performed. Also the possibility that the analysing light from the DRLFP set-up produced ketyls that stayed entrapped was excluded by performing ground state diffuse reflectance absorption spectra with our diffuse reflectance laser flash photolysis set-up. The ketyl radicals were only detected after laser pulse excitation.

In order to perform the LDA analysis, several decay curves in different instrumental timescales were recorded. A superposition of those decay traces was made by normalization of each decay curve at a time range where they overlap, in order to produce a composite decay with closely spaced data at short times and larger spaced values at long times. This procedure was adopted before (e.g. ref. 26,36,37) and is necessary because the abscissa is $\log t$, therefore a very large time range had to be used.

\section{Diffuse reflectance laser flash photolysis}

Time-resolved absorption spectra of samples of BZP/60 A silica, $\mathrm{BZP} / \mathrm{RP}-18$ and BZP/RP-8 complexes were obtained by the use of diffuse reflectance laser flash photolysis technique, developed by Wilkinson and co-workers. ${ }^{2-4}$ In this study the use of an intensified charged coupled device as detector allowed us to obtain timeresolved absorption spectra with nanometer spectral spacing., ${ }^{19-11}$ Fig. $3 \mathrm{a}, \mathrm{b}$ and $\mathrm{c}$ show the time-resolved absorption spectra of $250 \mu \mathrm{mol}$ of BZP per gram of the solid powdered substrate. Spectra $3 \mathrm{a}$ and $3 \mathrm{~b}$ were obtained for argon purged samples, exciting at $355 \mathrm{~nm}$, while spectra 3c were obtained after $266 \mathrm{~nm}$ excitation.

Diffuse reflectance laser flash photolysis provided us further experimental information of relevance in order to solve the problem of the identification of the species responsible for the low energy emission band peaking at $575 \mathrm{~nm}$ for the reverse phase silicas as adsorbents, as we will show now.

Transient absorption spectra of the BZP/60 A silica sample have shown the simultaneous formation of triplet benzophenone and also of hydroxylbenzophenone radical $\left(\mathrm{BZP}^{\cdot} \mathrm{OH}\right){ }^{20}$ as Fig. 3a shows. The triplet-triplet absorption spectra of microcrystalline BZP on a silica surface has been published a long time ago, ${ }^{4,29,37}$ but usually in a smaller wavelength range (400-700 $\mathrm{nm}$ or even smaller). The reason why we repeat the spectrum here is to show the importance of obtaining the transient absorption in a large spectral domain: at about $395 \mathrm{~nm}$ the dominant absorption on this surface is the $\mathrm{BZP} \cdot \mathrm{OH}$ radical absorption which remains even in the $20 \mathrm{~ms}$ timescale, i.e., after the complete disappearance of the BZP transient absorption.

The triplet-triplet absorption spectra of benzophenone was easily identified from comparison with the one published by Wilkinson and Willsher. ${ }^{4}$ The transient absorption which peaks at $395 \mathrm{~nm}$ can be assigned to $\mathrm{BZP} \cdot \mathrm{OH}$ radical by comparison with previously reported spectra in solution, ${ }^{38,39}$ or on the MCM-41 surface. $^{20}$

In part $b$ of Fig. 3, which shows the transient absorption spectra obtained after $355 \mathrm{~nm}$ excitation of BZP on the RP-18 surface, the $\mathrm{BZP} \cdot \mathrm{OH}$ radical absorption can be seen at about $395 \mathrm{~nm}$, but the dominant absorption is now peaking at 555 and $320 \mathrm{~nm}$, i.e. we have the ketyl radical from BZP transient absorption which lives 

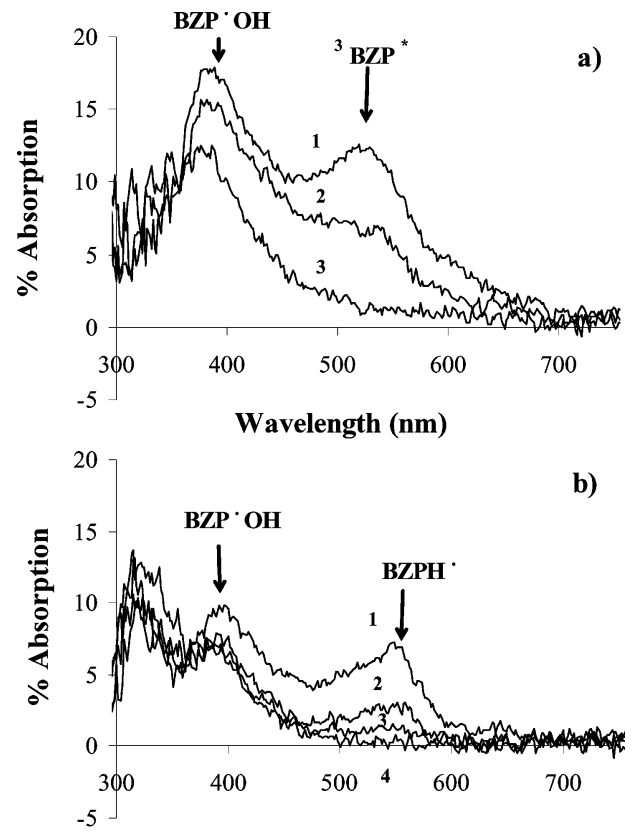

Wavelength (nm)

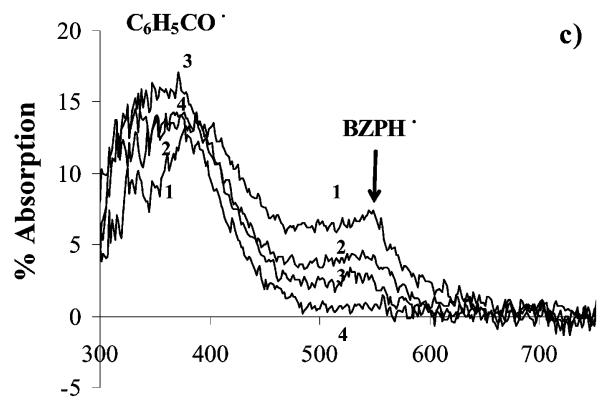

Wavelength (nm)

Fig. 3 Diffuse reflectance transient absorption spectra for argon purged samples of a) benzophenone adsorbed onto 60 Å pore silica, $250 \mu \mathrm{mol} \mathrm{g}{ }^{-1}$. $\left(\lambda_{\text {exc }}=355 \mathrm{~nm}\right)$. Curves 1 and 2 refer to 1 and $5 \mu$ s after laser pulse while curve 3 was obtained at $20 \mathrm{~ms}$. b) Benzophenone adsorbed onto reversed-phase silica (RP-18), $500 \mu \mathrm{mol} \mathrm{g}^{-1}$. $\left(\lambda_{\text {exc }}=355 \mathrm{~nm}\right)$. Curves 1,2 and 3 refer to 1, 5 and $20 \mu$ s after laser pulse while curve 4 was obtained at $20 \mathrm{~ms}$. c) Benzophenone adsorbed onto reversed-phase silica (RP-8), $250 \mu \mathrm{mol} \mathrm{g}^{-1}$. $\left(\lambda_{\text {exc }}=266 \mathrm{~nm}\right)$. Curves 1,2 and 3 refer to 0,1 and $5 \mu \mathrm{s}$ after laser pulse while curve 4 was obtained at $20 \mathrm{~ms}$.

longer than the triplet BZP, as shown in this Fig. 3. So now we have a double experimental evidence for the diphenylketyl radical: its fluorescence and its transient absorption spectra, both time resolved.

The interesting fact in Fig. 3c when compared to Fig. 3b, where $266 \mathrm{~nm}$ radiation was used to excite BZP adsorbed on RP-8 (similar results were obtained for RP-18, but those data are not shown) is that the benzoyl radical transient absorption ${ }^{11}$ is now well visible in the shorter wavelength range and at longer timescales. The identification could be made, again by comparison with previously published work, ${ }^{11}$ and this means that the BZP photochemistry is wavelength dependent, i.e. when using $266 \mathrm{~nm}$ laser excitation a much more rich photochemistry was obtained, which results, as we will see now in detail with the help of chromatographic separation and identification of products studies (GC-MS).

\section{Photodegradation products studies}

Lamp irradiation at $254 \mathbf{~ n m}$. The photoproducts were first studied under lamp irradiation $(254 \mathrm{~nm})$, in argon atmosphere and air equilibrated conditions, in all solid supports. As observed in previous photodegradation studies of $\mathrm{BZP},{ }^{9-11,20}$ 2hydroxybenzophenone (2-OHBZP) is the main photodegradation product for BZP on silica. The other isomers of hydroxybenzophenone $(m / z=198)$ and traces of compounds with $m / z=258$, assigned to phenylbenzophenones (PhBZP), were also found. In air equilibrated conditions benzoic acid is also one of the major degradation products, suggesting the involvement of molecular oxygen on its formation. 2-OHBZP and benzoic acid were also detected after lamp irradiation $(254 \mathrm{~nm})$ of $\mathrm{BZP} /$ calixarene complexes $^{9-11}$ and MCM-41 surface. ${ }^{20}$ The same photodegradation products were obtained on RP-18. Benzhydrol was also found on this support but is a minor degradation product in these conditions. The presences of $\mathrm{PhBZP}(\mathrm{s})$ indicate that BZP can undergo $\alpha$-cleavage. Since the $\mathrm{C}-\mathrm{CO}$ bond energy of benzophenone is higher than the excitation energy of the lowest triplet state, benzophenone in the $T_{1}$ state cannot dissociate. Therefore, as already reported in solution, ${ }^{40}$ this reaction must occur from a higher excited state and a wavelength dependence of the photoproduct distribution should in principle be found.

Laser irradiation at 355 and $266 \mathrm{~nm}$. A study of the photoproduct distribution was also made under $355 \mathrm{~nm}$ and $266 \mathrm{~nm}$ laser irradiation, in argon atmosphere. Fig. S2 of ESI $\dagger$ shows the photoproduct distribution on silica surface at $266 \mathrm{~nm}$ (part a) and $355 \mathrm{~nm}$ irradiation (part b). In what regards $266 \mathrm{~nm}$ irradiation, while under lamp irradiation 2-OHBZP is the main degradation product, in these conditions other isomers $(m / z=198)$ are formed in a significant amount. Products resulting from the $\alpha$ cleavage of BZP, namely biphenyl and $\operatorname{PhBZP}(\mathrm{s})(\mathrm{m} / \mathrm{z}=258)$, are formed in larger quantities than those obtained under lamp irradiation conditions. This result confirmed that this reaction pathway only takes place from a state higher in energy than that of $n \pi^{*}$ state. The photoproduct distribution also indicates that the $\alpha$-cleavage pathway is more important under laser than under lamp irradiation conditions. This result seems to indicate the importance of obtaining higher excited states of BZP, allowing therefore for a very efficient $\alpha$-cleavage reaction. It is also important to emphasise that benzhydrol was not detected on the silica surface.

Under $355 \mathrm{~nm}$ irradiation, 2-OHBZP and one of its isomers are the main degradation products on silica (see Fig. S2b of ESI). $\dagger$ All products derived from the $\alpha$-cleavage were not found in these irradiation conditions, confirming that the excitation to electronic states with higher energy than the $n \pi^{*}$ states are required for this $\alpha$-cleavage reaction to occur. Benzhydrol was also not detected in these irradiation conditions.

Fig. 4a shows the photoproduct distribution on RP-18 after $266 \mathrm{~nm}$ irradiation. As expected, 2-OHBZP and products resulting from the $\alpha$-cleavage are also present in this solid support. Given the reductive nature of RP-18, benzhydrol was also expected in this solid support. The used conditions did not allow for a good 

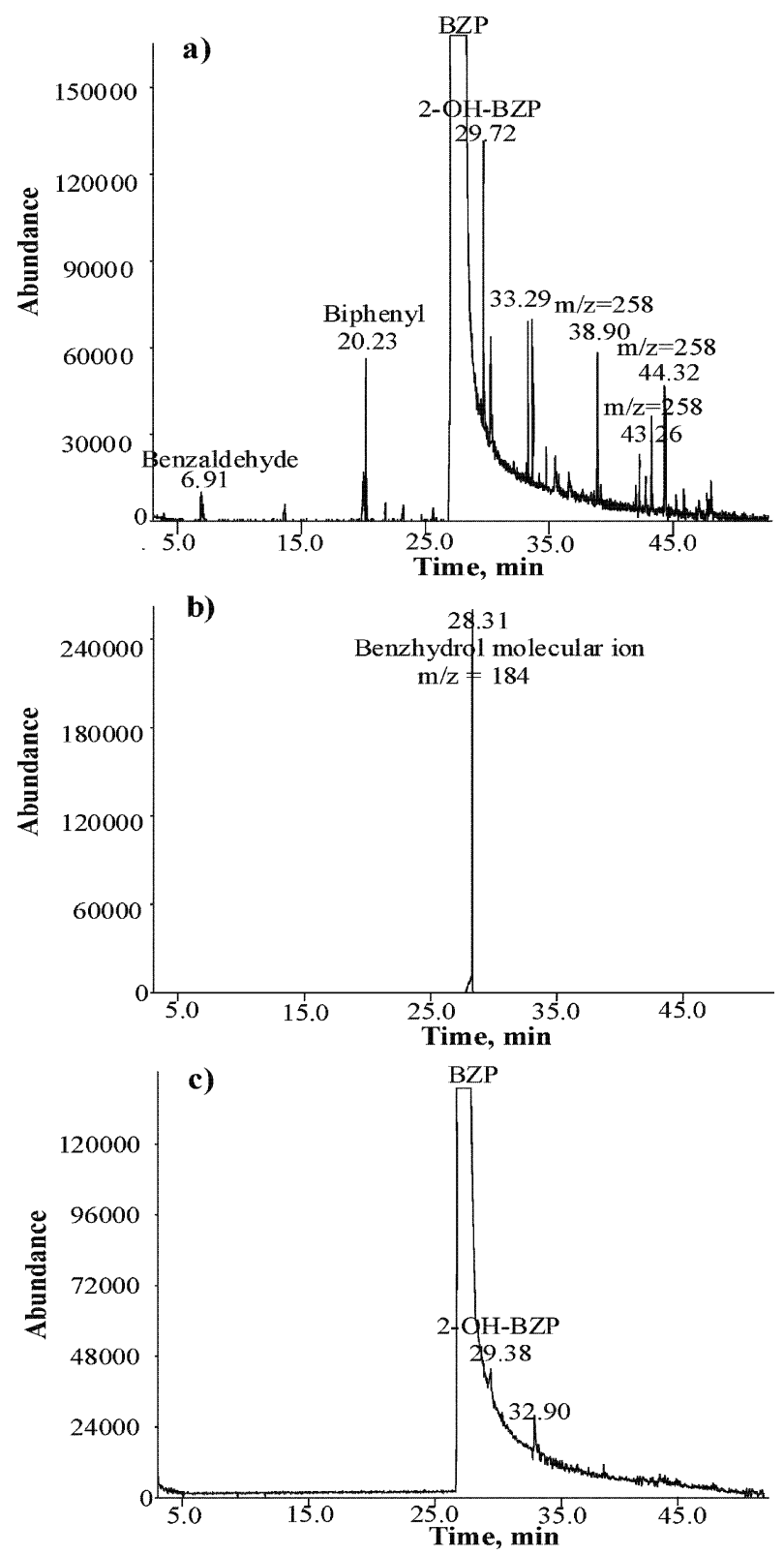

Fig. 4 Chromatographic results (GC-MS) of the extracts of benzophenone adsorbed onto reversed-phase silica (RP-18). With a) $266 \mathrm{~nm}$ excitation, b) $266 \mathrm{~nm}$ excitation with detection of the ion with $\mathrm{m} / \mathrm{z}=$ 184 and c) $355 \mathrm{~nm}$ excitation. Similar results were obtained for RP-8.

chromatographic separation of benzhydrol form BZP because the latter compound is present in much large concentration (the products were analysed at conversions lower than $5 \%$ ) and they show similar retention times. The single $\mathrm{m} / z=184$ ion chromatogram (the molecular ion of benzhydrol) showed that this compound is one of the major degradation products in this solid support (see Fig. 4b).

The results obtained on RP-18 in $355 \mathrm{~nm}$ indicated that 2OHBZP and benzhydrol, the latter detected in the same way as for $266 \mathrm{~nm}$ irradiation are the main photodegradation products (see Fig. 4c). Products showing mass spectra compatible with compounds with aliphatic structure were also detected in RP-18 at $266 \mathrm{~nm}$ (see Fig. 4a, products with retention time 33.29 and
$33.62 \mathrm{~min}$.) and at $355 \mathrm{~nm}$ (see Fig. 4c), product with retention time $32.90 \mathrm{~min}$. These products result from the reaction of the probe with the solid support, resulting namely from hydrogen abstraction and ketyl radical formation.

\section{Reaction pathways}

The obtained transient absorption and photoproduct data for BZP on silica and reversed-phase silica in the absence of oxygen can be explained considering three main reaction pathways: hydrogen atom abstraction from the adsorbent, which gives benzhydrol through the ketyl radical; $\alpha$-cleavage, which gives benzaldehyde, biphenyl and phenyl benzophenone isomers through benzoyl and phenyl radicals and photo hydrolysis, which gives 2hydroxybenzophenone through $\mathrm{BZP}^{\bullet} \mathrm{OH}$ radical formation (see Fig. 5).

Hydrogen abstraction is expected to be the main reaction pathway on RP-18 due to the reductive nature of this support. In fact, on this solid support the ketyl radical was detected by both time resolved luminescence and laser flash photolysis and benzhydrol was the main photodegradation product under all the studied conditions. Upon $266 \mathrm{~nm}$ irradiation the $\alpha$-cleavage of BZP emerges as another important degradation pathway. This primary photoreaction process is in agreement with the formation of benzaldehyde, biphenyl and phenyl benzophenone isomers, and explains the broadening of the transient absorption below $400 \mathrm{~nm}$ observed after $266 \mathrm{~nm}$ excitation. In fact the benzoyl radical was detected in the transient absorption studies and absorbs in this spectral region for all three adsorbents. This reaction was already reported ${ }^{20}$ and only occurs when a highly excited state of BZP is formed. This is in agreement with our results since both transient absorption and photodegradation products are compatible with this degradation pathway, which is only observed under $266 \mathrm{~nm}$ excitation, that promotes the $\pi \rightarrow \pi^{*}$ transition. Photo hydrolysis also occurs on the RP-18 surface. Both 2-OHBZP and its precursor $\left(\mathrm{BZP}^{\cdot} \mathrm{OH}\right)$ were detected, suggesting that in spite of the expected hydrophobic character of this surface, some water molecules must be present in order to allow this reaction. This $\mathrm{OH}$ adduct can result from the BZP radical cation, after reaction with water. ${ }^{41,42}$ The formation of the radical cation, according to ref. 7,41 and 42 , is due to an electron transfer process from the excited benzophenone to, in this case, the silica surface. ${ }^{7}$ However no absorption assignable to the radical cation transient was detected between $300 \mathrm{~nm}$ and $800 \mathrm{~nm}$, probably due to the low absorption in this spectral range and/or to its fast reaction, ${ }^{20,41}$ in a time-range which is not detectable in our set-up. In all three solids under study, we were not able to confirm this pathway for the $\mathrm{OH}$ adduct formation. However, the participation of water in this reaction pathway is a necessity.

On silica no data compatible with hydrogen abstraction were found. This result is in agreement with the low hydrogen donor ability of the silica surface. On this surface all data suggest that photo hydrolysis is the main degradation pathway. The detection of photoproducts and transient absorption compatible with the $\alpha$-cleavage was also observed on this solid support, under $266 \mathrm{~nm}$ irradiation. This is in agreement with the results obtained on RP18 and RP-8 and confirms that the photochemical behaviour of $\mathrm{BZP}$ is wavelength dependent. 


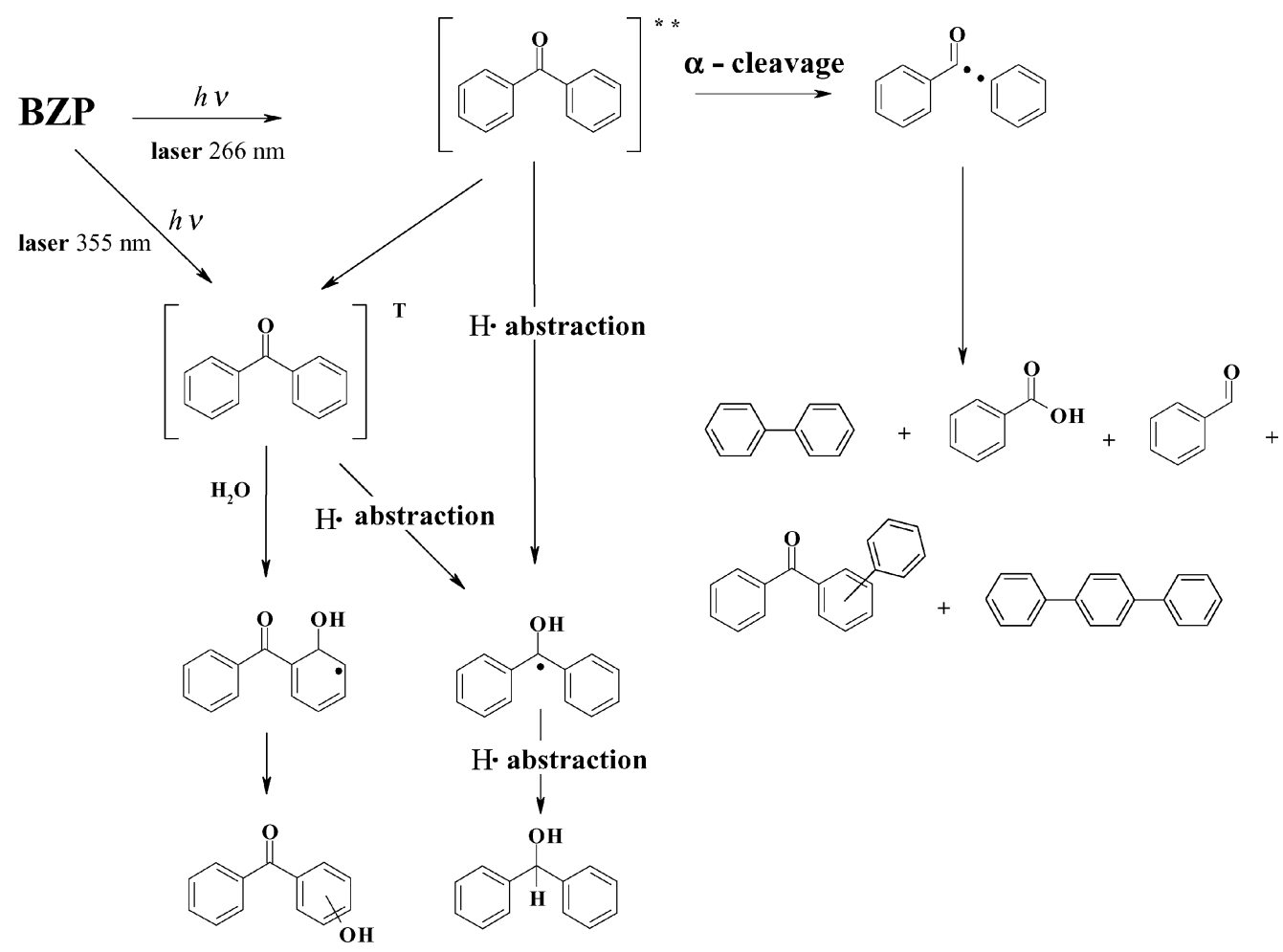

Fig. 5 Reaction mechanisms: benzophenone adsorbed onto 60 Å pore silica or reversed-phase silicas (RP-18 and RP-8).

\section{Conclusions}

BZP proved to be a very useful probe for the study of the reversed silica surfaces as compared to a simple porous silica surface, because it provided information regarding the polarity of these surfaces and also regarding the surface photochemical characteristics of these hosts.

The photochemistry of BZP onto porous silicas and reversedphase silicas is determined by the nature of the surface (silanols versus long alkyl chains). BZP phosphorescence, BZP ketyl radical fluorescence and hydrogen bonded BZP luminescence were detected, depending on the nature of the surface.

A lifetime distributions analysis (LDA) provided important information regarding the adsorption sites of BZP on $60 \AA$ pore silica and also revealed an important quenching effect on the case of reversed silica.

Diffuse reflectance transient absorption spectra revealed the presence of the triplet state of BZP in all supports under study, but also of the diphenylketyl radical, $\mathrm{BZP} \cdot \mathrm{OH}$ radical, benzoyl radical and other transient species, depending on the surface and on the energy of the excitation radiation.

The photochemistry of BZP on surfaces is in fact wavelength dependent: with $266 \mathrm{~nm}$ excitation $\alpha$-cleavage reaction predominates and a variety of photoproducts was obtained and identified by GC-MS, while with $355 \mathrm{~nm}$ excitation only BZP ketyl radical and/or OHBZP formation was detected.

\section{Acknowledgements}

T.J.F.B. thanks FCT for Doctoral fellowship SFRH/BD/ $8143 / 2002$. J.P.S. thanks FCT for a Post-Doctoral fellowship
SFRH/BPD/15589/2001 and I.F.M. thanks FSE for financial support.

\section{References}

1 A. M. Botelho do Rego and L. F. Vieira Ferreira, in Handbook of Surfaces and Interfaces of Materials, ed. H. S. Nalwa, Academic Press, San Diego, 2001, vol. 2, ch. 7, pp. 275-313.

2 F. Wilkinson and G. P. Kelly, in Photochemistry on Solid Surfaces, ed. M. Anpo and T. Matsuara, Elsevier, Amsterdam, 1989, pp. 31-47.

3 F. Wilkinson and G. P. Kelly, in Handbook of Organic Photochemistry, ed. J. C. Scaiano, CRC Press, Boca Raton, 1989, vol. 1, ch. 12, pp. 293-314.

4 F. Wilkinson and C. J. Willsher, Detection of triplet-triplet absorption of microcrystalline benzophenone by diffuse-reflectance laser flash photolysis, Chem. Phys. Lett., 1984, 104, 272-276.

5 J. C. Scaiano and H. Garcia, Intrazeolite Photochemistry: toward supramolecular control of molecular photochemistry, Acc. Chem. Res., 1999, 32, 783-793.

$6 \mathrm{~S}$. Hashimoto, Zeolite photochemistry: impact of zeolites on photochemistry and feedback from photochemistry to zeolite science, J. Photochem. Photobiol., C: Photochem. Rev., 2003, 4, 19-49.

7 J. K. Thomas, Physical Aspects of Radiation-Induced Processes on $\mathrm{SiO}_{2}, \gamma-\mathrm{Al}_{2} \mathrm{O}_{3}$, Zeolites and Clays, Chem. Rev., 2005, 105, 1683-1734.

$8 \mathrm{~J}$. K. Thomas, Effect of $\mathrm{SiO}_{2}$ and zeolite surfaces on the excited triplet state of benzophenone: a spectroscopic and kinetic study, Photochem. Photobiol. Sci., 2004, 3, 483-488.

9 L. F. Vieira Ferreira, M. R. Vieira Ferreira, J. P. Da Silva, I. Ferreira Machado, A. S. Oliveira and J. V. Prata, A novel laserinduced luminescence resulting from benzophenone $/ O$-propylated $p$ tert-butylcalix[4]arenes complexes. A diffuse reflectance study, Photochem. Photobiol. Sci., 2003, 2, 1002-1010.

10 L. F. Vieira Ferreira, M. R. Vieira Ferreira, A. S. Oliveira, T. J. F. Branco, J. V. Prata and J. C. Moreira, Diffuse reflectance studies of $\beta$-phenylpropiophenone and benzophenone inclusion complexes with calix[4], [6] and [8] arenes, Phys. Chem. Chem. Phys., 2002, 4, 204-210.

11 L. F. Vieira Ferreira, I. Ferreira Machado, A. S. Oliveira, M. R. Vieira Ferreira, J. P. Da Silva and J. C. Moreira, A diffuse reflectance 
comparative study of benzil inclusion within $p$-tert-butylcalix $[n]$ arenes ( $n=4,6$ and 8) and silicalite, J. Phys. Chem. B, 2002, 106, 12584 12593.

12 L. F. Vieira Ferreira, J. C. Netto-Ferreira, I. Khmelinskii, A. R. Garcia and S. M. B. Costa, Photochemistry on surfaces: matrix isolation mechanisms for study of interactions of benzophenone adsorbed on microcrystalline cellulose investigated by diffuse reflectance and luminescence techniques, Langmuir, 1995, 11, 231236.

13 L. F. Vieira Ferreira, M. R. Freixo, A. R. Garcia and F. Wilkinson, Photochemistry on surfaces: fluorescence quantum yield determination of dyes adsorbed on microcrystalline cellulose, J. Chem. Soc., Faraday Trans., 1992, 88, 15-22.

14 L. F. Vieira Ferreira, A. R. Garcia, M. R. Freixo and S. M. B. Costa, Photochemistry on surfaces: the solvent-matrix effect on the swelling of cellulose. An emission and absorption study of adsorbed auramine-O, J. Chem. Soc., Faraday Trans., 1993, 89, 1937-1944.

15 L. F. Vieira Ferreira, M. R. Vieira Ferreira, A. S. Oliveira and J. C. Moreira, Potentialities of diffuse reflectance laser-induced techniques in solid phase: a comparative study of benzophenone inclusion within $p$-tert-butylcalixarenes, silicalite and microcrystalline cellulose, J. Photochem. Photobiol., A, 2002, 153, 11-18.

16 L. F. Vieira Ferreira, A. S. Oliveira and J. C. Netto-Ferreira, in Fluorescence Microscopy and Fluorescent Probes 3, ed. A. Kotyc, Espero Publishing, Prague, 1999, pp. 199-208.

17 J. P. Da Silva, L. F. Vieira Ferreira, A. M. Da Silva and A. S. Oliveira, A comparative study of the photophysics and photochemistry of 4chlorophenol adsorbed on silicalite and $\beta$-cyclodextrin, J. Photochem. Photobiol., A, 2002, 151, 157-164.

18 L. F. Vieira Ferreira, M. J. Lemos, M. J. Reis and A. M. Botelho do Rego, UV/Vis absorption and luminescence and X-ray photoelectron spectroscopic studies of rhodamine dyes adsorbed onto different pore size silicas, Langmuir, 2000, 16, 5673-5680.

19 B. Q. Ma, L. F. Vieira Ferreira and P. Coppens, Structure and photoluminescence of a benzil nanocolumn in a $C$-methylcalix[4]resorcinarenebased framework, Org. Lett., 2004, 6, 1087-1090.

20 J. P. Da Silva, I. Ferreira Machado, J. P. Lourenço and L. F. Vieira Ferreira, Photochemistry of benzophenone adsorbed on MCM-41 surface, Microporous Mesoporous Mater., 2005, 84, 1-10.

21 D. A. Skoog and D. M. West, in Analytical Chemistry: An introduction, Saunders College Pub., Philadelphia, 4th edn, 1986, ch. 18.

22 J. P. Da Silva, A. M. Da Silva, I. V. Khmelinskii, J. M. G. Martinho and L. F. Vieira Ferreira, Photophysics and Photochemistry of Azole Fungicides Triadimefon and triadimenol, J. Photochem. Photobiol., A, 2001, 142, 31-37.

23 J. P. Da Silva and L. F. Vieira Ferreira, Surface photochemistry of pesticides: an approach using diffuse reflectance and chromatographic techniques, Environ. Sci. Technol., 2004, 38, 2849-2856.

24 N. J. Turro, Modern Molecular Photochemistry, Benjamin Cummings, Menlo Park, CA, 1978.

25 T. J. F. Branco, A. M. Botelho do Rego, I. Ferreira Machado and L. F. Vieira Ferreira, A luminescence lifetime distributions analysis in heterogeneous systems by the use of Excel's Solver, J. Phys. Chem. B, 2005, 109, 15958-15967.
26 J. C. Scaiano, M. Kaila and S. Corrent, Intrazeolite Photochemistry. 19. Effect of the "spectator" pyridine on the behavior of the carbonyl triplet states in the zeolite NaY, J. Phys. Chem. B, 1997, 101, 8564-8568.

27 M. Barra and J. C. Scaiano, Photoinduced transient phenomena in cyclodextrin solid complexes: photochemistry of aromatic ketones, Photochem. Photobiol., 1995, 62, 60-64.

$28 \mathrm{M}$. Barra and K. A. Agha, Application of diffuse laser-flash photolysis to the study of aromatic probes in $\beta$-cyclodextrin solid complexes, J. Photochem. Photobiol., A, 2002, 109, 293-298.

29 N. J. Turro, Photochemistry of Ketones adsorbed on Porous Silica, Tetrahedron, 1987, 43, 1589-1616.

30 J. C. Netto-Ferreira and J. C. Scaiano, Photochemistry and photophysics from the excited states of diaryl ketyl radicals, Res. Chem. Intermed., 1989, 12, 187-201.

31 K. R. Naqvi and U. P. Wild, Room temperature fluorescence of the diphenyl ketyl radical, Chem. Phys. Lett., 1976, 41, 570-574.

32 M. R. Topp, Biphotonic excitation of the fluorescence of benzophenone ketyl, Chem. Phys. Lett., 1976, 39, 423-426.

33 H. L. Casal and J. C. Scaiano, Intrazeolite photochemistry. I. Phosphorescence enhancement of aromatic ketones included in silicalite, Can. J. Chem., 1984, 62, 628-629.

34 G. Scharf and J. D. Winefordner, Phosphorescence characteristics of acetophenone, bemzophenone, $p$-aminobenzophenone and Michler's ketone in various environments, Talanta, 1986, 33, 17-25.

35 R. W. Redmond and J. C. Scaiano, A two-laser technique for the evaluation of the quantum yields of fluorescence from excited reaction intermediates. The case of diphenylketyl radicals, Chem. Phys. Lett., 1990, 166, 20-25.

36 G. Kelly, C. J. Willsher, F. Wilkinson, J. C. Netto-Ferreira, A. Olea, D. Weir, L. J. Johnston and J. C. Scaiano, Intrazeolite photochemistry. VI. Diffuse reflectance laser flash photolysis and product studies of diphenylmethyl radicals on solid supports, Can. J. Chem., 1990, 68, 812-819.

37 S. Kazanis, A. Azarani and L. J. Johnston, Diffuse reflectance laser flash photolysis studies of reactions of triplet benzophenone with hydrogen donors on silica, J. Phys. Chem., 1991, 95, 4430-4435.

38 M. B. Ledger and G. Porter, The photochemistry of aromatic carbonyl compounds in aqueous solution, J. Chem. Soc., Faraday Trans., 1972, 68, 539-553.

39 S. B. Sharma, M. Mudaliar, B. S. M. Rao, H. Mohan and J. P. Mittal, Radiation chemistry oxidation of benzaldehyde acetophenone and benzophenone, J. Phys. Chem. A, 1997, 101, 8402-8408.

40 Y. Kajii, T. Suzuki, Y. Takatori, K. Shibuya and K. Obi, Photodissociation of Higly-Excited Triplet State of Benzophenone Studied by a Time-Resolved Thermal Lensing Technique, Bull. Chem. Soc. Jpn., 1992, 65, 1349-1355.

41 S. Baral-Tosh, S. K. Chattopadhyay and P. K. Das, A laser flash photolysis study of paraquat reduction by photogenerated aromatic ketyl radicals and carbonyl triplets, J. Phys. Chem., 1984, 88, 1404 1408.

42 F. Elisei, G. Favaro and H. Gorner, Ion-forming processes on $248 \mathrm{~nm}$ laser excitation of benzophenone in aqueous solution: a time-resolved absorption and conductivity study, J. Photochem. Photobiol., A, 1991, 59, 243-253. 